\title{
Application of a Computer-aid Diagnosis of Pneumoconiosis for CR X-ray Images
}

\author{
${ }^{1}$ Koji Abe, ${ }^{2}$ Masahide Minami, ${ }^{1}$ Ryosuke Miyazaki and ${ }^{3}$ Haiyan Tian \\ ${ }^{1}$ Interdisciplinary Graduate School of Science and Engineering, Kinki University, Japan; \\ ${ }^{2}$ Kanazawa Gakuin University, Japan; \\ ${ }^{3}$ Graduate School of Engineering, Kobe University, Japan; \\ koji@info.kindai.ac.jp; saboten0078@yahoo.co.jp; maminami@dream.com; \\ tian.haiyan2006@gmail.com
}

\begin{abstract}
This paper presents a method for applying a computer-aided diagnosis (CAD) for pneumoconiosis to chest X-ray images digitalized by the computed radiography (CR) system. When we reported the CAD before, we showed performance of the CAD for chest X-ray images digitalized by a CCD scanner. However, since density distribution of the CR X-ray images is quite different from the images digitalized by CCD scanner, it is necessary to equip some preprocessing into the CAD for applying to the CR images. In this paper, as the first trial for the application, drawing rib edges and additional lines on the $C R$ images by a tablet PC, we examined whether the CAD can be interactively applied to the CR images. Using 51 chest CR X-ray images, we compared the proposed CAD system with the existing system for the images digitalized by CCD scanner.
\end{abstract}

Keywords: Computer-aided diagnosis; Pneumoconiosis; Chest X-ray image; Medical image processing.

\section{Introduction}

Pneumoconiosis is interstitial lung disease caused by inhalation of fine particles (e.g., coal pneumoconiosis). In recent years, in addition to coal workers, dental technicians also suffer from the disease. However, since it is difficult for even expert diagnosticians on pneumoconiosis to diagnose pneumoconiosis, disagreement between diagnosticians is often happened. Besides, the experts teach techniques on the diagnosis to immature diagnosticians based on just their experience. For the reasons, computer-aided diagnosis (CAD) systems for pneumoconiosis have been required as a second opinion for diagnosticians. CAD systems for pneumoconiosis have been reported since 1970s [1-4]. Their measurements of abnormalities for pneumoconiosis broadly are two ways: the one measures the abnormalities based on texture analysis [1,2], another one extracts small round opacities and measures their size and number as well as the real diagnosis by diagnosticians [3, 4]. All the systems were proposed to images obtained by a custom-made scanner (e.g., a drum scanner or a film scanner). Therefore, if the systems were applied in general clinics, the clinics would have to deploy the special scanner, or order scanning chest radiographs to a printing company in spite of high costs. 
In order to enhance cost-performance of CAD for pneumoconiosis, a CAD system for pneumoconiosis using images digitalized by a common CCD scanner was reported $[5,6]$. This system is composed with a CCD scanner and a tablet PC, and discriminates pneumoconiosis with abnormalities in rib areas. Since the images obtained from X-ray pictures by a CCD scanner are extremely unclear and it is hard to extract rib areas automatically, in this system, the user draws rib edges on the images manually using the tablet PC. The reason why only X-ray pictures are used in diagnosis of pneumoconiosis is because there is a criterion that diagnosticians have to diagnose pneumoconiosis comparing $X$-ray pictures with the standard X-ray pictures of pneumoconiosis provided by the International Labour Organization (ILO). Similarly, in Japan, the Ministry of Health, Labour and Welfare provided another set of the standard Xray pictures. And, both of the ILO and the ministry opened new standard images in 2011 [7]. Both of the new standard sets are digital images digitalized by the computed radiography (CR) system. Since a picture digitalized by $C R$ is saved as a digital file, CR enables diagnosticians to read X-ray pictures on a monitor. CR has been already regular radiography in Japan.

With the revision of the standard pictures from X-ray pictures to CR X-ray images, this paper examines whether the prior CAD system for pneumoconiosis using the X-ray images digitalized by a CCD scanner can apply to chest CR X-ray images. Basically, X-ray images digitalized by CCD scanner are unclear. On the other hand, since the shadows in the CR images are appeared clearly, density distribution of the CR image is quite different from the X-ray image digitalized by a CCD scanner. Hence, if we consider to apply the CAD to the $C R$ images, we need to customize the CAD equipping some pre-processing. Therefore, in order to apply the CAD to the CR images, this paper proposes a manual pre-processing method of drawing rib edges and additional lines on the CR images before the diagnosis by the CAD. If the system can apply to either of chest CR X-ray images and images digitalized by CCD scanner, the system is useful in the diagnosis for either of them at a low cost.

\section{Pneumoconiosis Classification}

The level of pneumoconiosis was indicated by profusion of small round opacities, where categories 0-3 were established. Figure 1 shows samples of the standard images provided by the Japanese ministry. Normal cases belong to category 0 , where the opacities are not observed visually. And, abnormal ones belong to category 1,2 , or 3 , where the most serious level is category 3 and the opacities are observed most in category 3. Although diagnosticians diagnose pneumoconiosis comparing chest $\mathrm{X}$-ray pictures with the standard pneumoconiosis images prepared in every category, their own experience much depends on the diagnosis. For example, gray levels at the opacities are very similar to intersections of vascular shadows. 


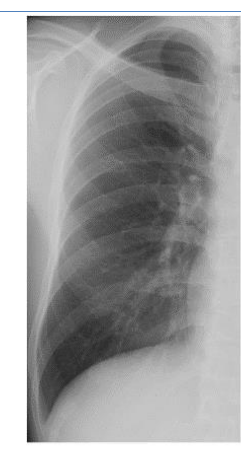

category 0

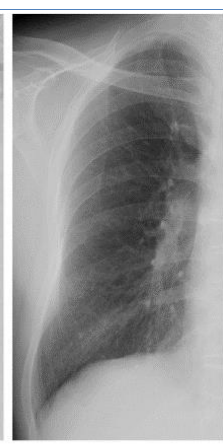

category 1

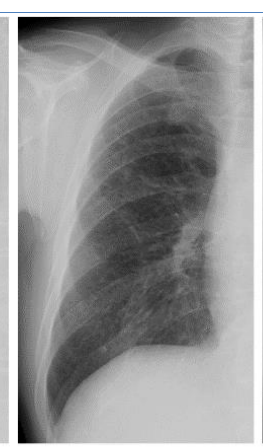

category 2

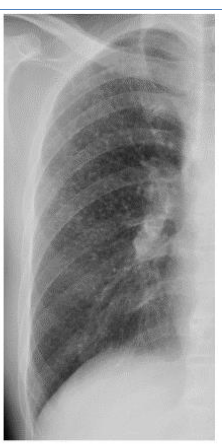

category 3

Figure 1: Standard images of pneumoconiosis

(Provided by the Ministry of Health, Labour, and Welfare in Japan).

In Japan, the criteria of the classification are defined under the Japanese Pneumoconiosis Law in accordance with criteria of pneumoconiosis in the ILO.

\section{CAD for Pneumoconiosis X-ray Images Digitalized by CCD Scanner}

\subsection{Overview}

In the CAD system for pneumoconiosis X-ray images digitalized by CCD scanner [6], abnormalities of pneumoconiosis are measured by extracting characteristics of the density distribution in rib areas. The rib areas are manually designated using a tablet PC. First, the right lung area in chest X-ray pictures is digitalized by a CCD scanner. Next, the chest image is displayed on the tablet PC and the user draws curves along the edges of rib shadows on the image using the tablet PC. And then, the rib areas are designated by the drawn curves. Finally, abnormalities of pneumoconiosis are extracted from the rib areas. The extracted abnormalities are used as valuables for discrimination of the X-ray images into normal or abnormal cases in pneumoconiosis.

\subsection{Preprocessing}

The right lung area in chest X-ray pictures $(35 \mathrm{~cm} \times 35 \mathrm{~cm})$ is digitalized by a CCD scanner. The chest Xray image is configured with $300 \mathrm{dpi}$ and 256 gray levels. Next, the image is resized into 1000 pixels in height without changing the aspect ratio. And then, the range of the gray value in the image is standardized by the linear histogram stretching.

\subsection{Drawing of Rib Edges on the X-ray Image with Tablet PC and Extraction of Rib Areas}

The image is displayed on the tablet PC and the user draws 8 curves with white color along the edges of 4 ribs on the image using the tablet PC. The manual for drawing the curves is designed as below.

[Manual for drawing the rib edges]

1. Open the right lung image in a paint tool.

2. Select the round brush and set the thickness of the circle as 4 pixels.

3. Select "white" to the line color.

4. Look at the curve shown in Figure 2(b) as the guide of start points for drawing rib edges. 
5. Imaging the curve on the image by yourself, decide the start point for the drawing and start drawing the curve along an edge of a rib.

6. Stop drawing the curve at a location where the edge cannot be seen.

7. Until you finish drawing all couples of the edges for 4 ribs, repeat 5 and 6.

As shown in 4 and 5, Figure 2(b) is shown to the user as the guide for aiming at the location of the start point for the drawing. Figure 2(c) shows an example of the curves drawn to Figure 2(a). Then, as shown in Figure 2(d), the rib areas $R_{1}-R_{4}$ are extracted by the drawn edges (Refer to [6]).

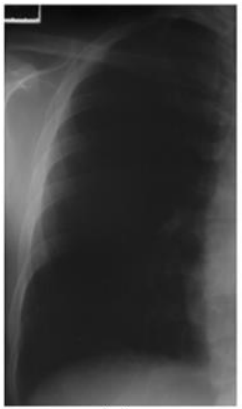

(a)

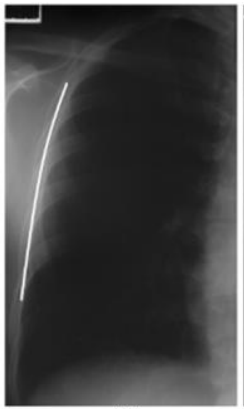

(b)

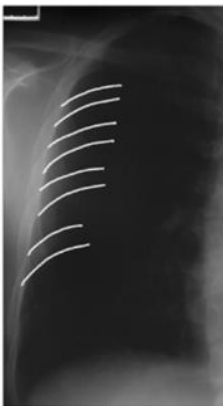

(c)

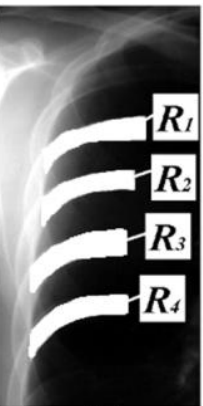

(d)

Figure 2: Drawing of rib edges according to the manual and rib areas $R_{1}-R_{4}$ extracted from the edges.

As shown in Figure 1 and Figure 2, we can see that the images digitalized by CCD scanner are extremely unclear than CR images.

\subsection{Extraction of Abnormalities and Discrimination of Pneumoconiosis}

Figure 3 shows a rib area $R_{m}$ extracted above, where the first line is regarded as $k$-th scanning line $(k=1)$ and the value $k$ is added by 1 whenever the scanning line is shifted to the right by 1 pixel. Besides, the uppermost pixel on $k$-th scanning line is regarded as $j=1$ and the value $j$ is added by 1 whenever the pixel goes down by 1 pixel. The linear histogram stretching is applied to every scanning line. In the scanning on the $k$-th scanning line, all the pixels on the line are divided into the upper set and the lower set. The boundary of them is obtained by the discriminant analysis for the pixels. In regarding $t_{m}[k]$-th pixel counted from the uppermost edge as the boundary $t_{m}[k]$, the upper set is composed of pixels of $j=$ $1 \sim t_{m}[k]-1$ and the lower set is $j=t_{m}[k]+1 \sim h e i R_{m}[k]$.

As the scanning is conducted from the upper edge to the lower edge along the scanning line regarding $v[j]$ as the gray value of the $j$-th scanning spot, by using the parameters shown in Figure 3 , the abnormality $A b n\left(R_{m}\right)$ of $k$-th scanning line in $R_{m}$ is defined as

$$
\operatorname{Abn}\left(R_{m}[k]\right)=\left[\frac{1}{h e i R_{m}[k]-1}\left(\sum_{j=1}^{h e i R_{m}[k]}|v[j]-\operatorname{avg}[k, j]|^{2}\right)\right]^{\frac{1}{2}}
$$

where 


$$
\operatorname{avg}[k, j]=\left\{\begin{array}{l}
\text { if } 1 \leq j \leq t_{m}[k],\left(\sum_{i=1}^{t_{m}[k]-1} v[j]\right) /\left(t_{m}[k]-1\right) \\
\text { if } j=t_{m}[k], v[j] \\
\text { if } t_{m}[k]<j \leq h e i R_{m}[k],\left(\sum_{i=t_{m}[k]+1}^{h e i R_{m}[k]} v[j]\right) /\left(h e i R_{m}[k]-t_{m}[k]\right)
\end{array}\right.
$$

Next, shifting the scanning line in $R_{m}$ shifts one by one to the scanning line at the most right side horizontally, the abnormality in $R_{m}, A b n\left(R_{m}\right)$ is defined as

$$
\operatorname{Abn}\left(R_{m}\right)=\frac{1}{w_{i d R_{m}}}\left(\sum_{k=1}^{w i d R_{m}} A b n\left(R_{m}[k]\right)\right)
$$

where wid $_{m}$ is the number of the horizontal shifts. Then, an abnormality in the whole rib areas $A b n R$ is defined as

$$
A b n R=\frac{1}{L_{R}}\left(\sum_{m=1}^{4} \sum_{k=1}^{w i d R_{m}} A b n\left(R_{m}[k]\right)\right)
$$

where

$$
L_{R}=\sum_{m=1}^{4} w i d R_{m}
$$

Besides, the maximum value $A b n R M A X$ in $A b n\left(R_{m}\right)(m=1 \sim 4)$ is represented as another abnormality.

$$
A b n R M A X=\max \left\{\operatorname{Abn}\left(R_{1}\right), A b n\left(R_{2}\right), A b n\left(R_{3}\right), A b n\left(R_{4}\right)\right\}
$$

Thus, the abnormality $A b n R$ represents an overall abnormality of pneumoconiosis in rib areas and $A b n R M A X$ represents local abnormalities of pneumoconiosis in all the rib areas.

After the extraction of the abnormalities, regarding the abnormalities as variables for discriminant machines, the diagnosis of pneumoconiosis is conducted by discriminating between normal cases and abnormal cases.

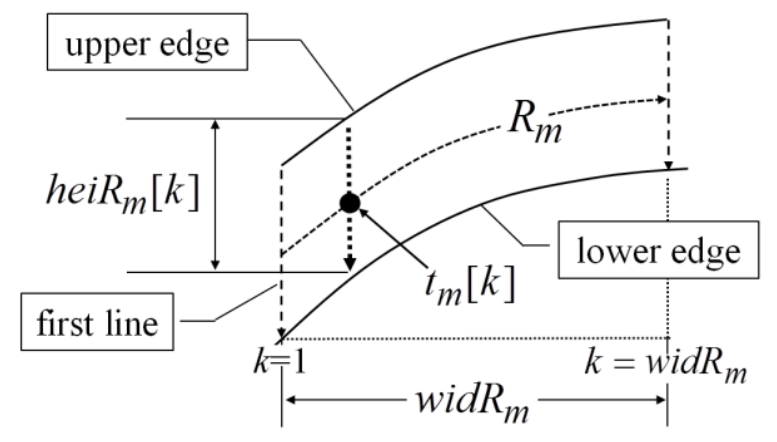

Figure 3: A rib $R_{m}$ and parameters used in the equations (3)-(5). 


\section{Customization of the Drawing Manual for Chest CR X-ray images}

Figure 4(b) shows a result that a user drew the rib edges to Figure 4(a) according to the manual shown in Sect. 3. Thus, differing from the case of the unclear images digitalized by CCD scanner, the user can confidently draw the edges on the whole ribs. However, due to the drawing, the extracted rib areas would contain the clavicular shadow or the lung marking (a mass of linear shadows appeared in the lung area of chest X-ray images) and they could become noises in extracting the abnormalities from the rib areas. Therefore, to draw the edges on the CR images without containing them, the manual is customized for the CR images as below.

[Manual for drawing the rib edges (for CR X-ray images)]

1. Open the right lung image in a paint tool.

2. Select the round brush and set the thickness of the circle as 7 pixels.

3. Draw a curve with a color except white along the lower edge of the clavicular shadow (as (A) in Figure 4(c)).

4. Draw a curve with a color except white at left side of the lung marking and separate the lung area into the lung marking and outside of the lung area (as (B) in Figure 4(c)).

5. Draw 8 curves along 4 couples of rib edges with white from the boundary between the lung area and its outside (i.e., left side contour of the right lung) until reaching either of the curves drawn in 3 and 4 as shown in Figure 4(c).

Figure 4(d) shows the rib areas extracted from Figure 4(c).

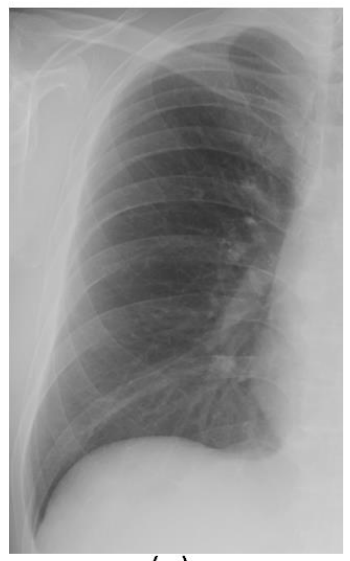

(a)

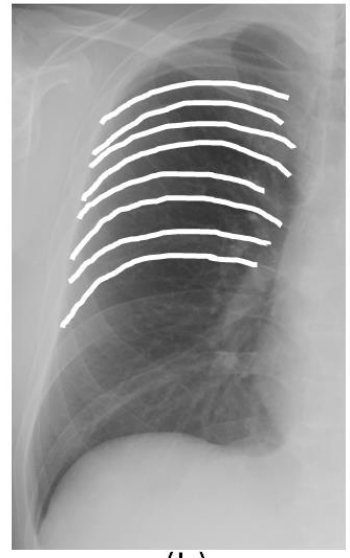

(b)

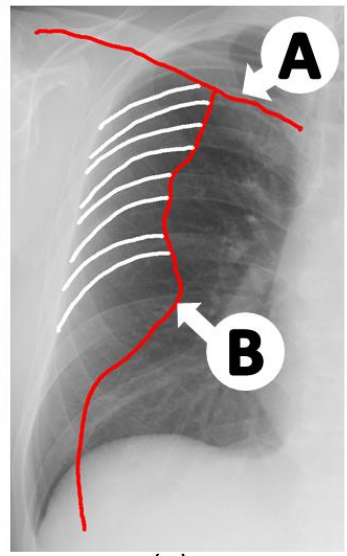

(c)

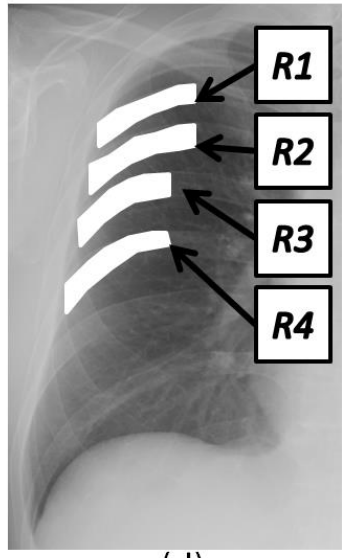

(d)

Figure 4: Drawing of the rib edges ((a): original image (b): drawing by the old manual, (c): drawing by the customized manual), and (d) is the rib areas $R_{1}-R_{4}$ extracted from image (c).

\section{Experimental Results}

The abnormalities were extracted from 51 chest CR X-ray images (resolution: 300 dpi, gray level: 8 bits, format: bitmap image). The number of images which belong in category 0 (normal case) is 36 , the number in category 1 is 6 , the number in category 2 is 6 and the number in category 3 is 3 . All the abnormal cases are the standard image of pneumoconiosis provided by the Japanese ministry. To obtain 
Koji Abe, Masahide Minami, Ryosuke Miyazaki and Haiyan Tian; Application of a Computer-aid Diagnosis of Pneumoconiosis for CR X-ray Images, Journal of Biomedical Engineering and Medical Imaging, Volume 1, No 5, Oct (2014) ,pp 113-122

the rib areas, three non-experts (represented as userA, userB, and userC) in pneumoconiosis diagnosis drew the rib edges on all the images with a tablet PC (ThinkPad 200Tablet 4184-F5J). The total time used for drawing all the images was about 60 minutes in every user. Table 1 shows the mean and standard deviation (SD) of $A b n R$ and AbnRMAX extracted from each of the normal and abnormal cases. From Table 1, we can see both of two abnormalities in abnormal cases are higher than normal cases, and the abnormalities are appropriately extracted for the discrimination of pneumoconiosis in both of the proposed methods.

Table 1: The mean value and the standard deviation of the abnormalities in the proposed method.

\begin{tabular}{|c|l|c|c|c|c|}
\hline \multirow{2}{*}{ Abnormalities } & \multirow{2}{*}{ User } & \multicolumn{2}{|c|}{ normal } & \multicolumn{2}{c|}{ Abnormal } \\
\cline { 3 - 6 } & & mean & SD & Mean & SD \\
\hline \multirow{4}{*}{ AbnR } & userA & 2.3 & 0.2 & 3.1 & 0.5 \\
\cline { 2 - 6 } & userB & 2.2 & 0.2 & 3.0 & 0.4 \\
\cline { 2 - 6 } AbnRMAX & userC & 2.1 & 0.2 & 3.0 & 0.5 \\
\hline \multirow{3}{*}{} & userA & 2.5 & 0.3 & 3.5 & 0.6 \\
\cline { 2 - 6 } & userB & 2.5 & 0.3 & 3.5 & 0.5 \\
\cline { 2 - 6 } & userC & 2.4 & 0.3 & 3.3 & 0.5 \\
\hline
\end{tabular}

Next, we examine performance of the proposed method from the discrimination of the images into normal or abnormal (i.e. pneumoconiosis) cases by regarding the abnormalities as variables for the discriminations. As the way of the discrimination, Random Trees (RT), a Neural Network (NN), a linear Support Vector Machine (SVM) were applied. Training set and test set were chosen by the leave-one-out cross validation [8] in all the discriminations. In fact, every discrimination was conducted as the following procedure:

1. Choose one image from all the images as test data, and use the other images as training set.

2. Discriminate the test data between normal and abnormal cases.

3. Repeat the procedure from 1 to 2 to every combination changing the test data.

Table 2 shows the recall (Rec.) and the precision (Pre.) in the discriminations. Rec. and Pre. are defined as

$$
\begin{aligned}
& \text { Rec. }=\frac{X_{h \cap c}}{X_{h}} \times 100 \\
& \text { Pre. }=\frac{X_{h \cap c}}{X_{c}} \times 100
\end{aligned}
$$

where $X$ represents the number of images, $X_{h}$ is the number of the correct answers, and $X_{c}$ is the number of images discriminated by the proposed method. Table 2 shows the ratios have been more than $80 \%$ except one part (Rec. of abnormal case in NN by userA). 
Table 2: Experimental results of the discrimination of pneumoconiosis by the proposed method.

\begin{tabular}{|c|c|c|c|c|c|c|c|}
\hline \multirow{2}{*}{ User } & \multirow{2}{*}{ Case } & \multicolumn{2}{|c|}{ RT } & \multicolumn{2}{c|}{ NN } & \multicolumn{2}{c|}{ SVM } \\
\cline { 3 - 8 } & & Rec. & Pre. & Rec. & Pre. & Rec. & Pre. \\
\hline \multirow{2}{*}{ userA } & Normal & $97.2 \%$ & $92.1 \%$ & $97.2 \%$ & $89.7 \%$ & $91.7 \%$ & $94.3 \%$ \\
\cline { 2 - 8 } & Abnormal & $80.0 \%$ & $92.3 \%$ & $73.3 \%$ & $91.6 \%$ & $86.7 \%$ & $81.3 \%$ \\
\hline \multirow{2}{*}{ userB } & Normal & $91.6 \%$ & $94.2 \%$ & $94.4 \%$ & $97.1 \%$ & $88.8 \%$ & $100 \%$ \\
\cline { 2 - 8 } & Abnormal & $86.6 \%$ & $81.2 \%$ & $93.3 \%$ & $87.5 \%$ & $100 \%$ & $78.9 \%$ \\
\hline \multirow{2}{*}{ userC } & Normal & $91.7 \%$ & $97.0 \%$ & $94.4 \%$ & $97.2 \%$ & $94.4 \%$ & $97.2 \%$ \\
\cline { 2 - 8 } & Abnormal & $93.2 \%$ & $82.4 \%$ & $93.2 \%$ & $87.5 \%$ & $93.2 \%$ & $87.5 \%$ \\
\hline
\end{tabular}

\section{Discussions}

Figure $7(a)(b)(c)$ show the distribution maps of all the data by the abnormalities, where $\bigcirc$ is normal case and $X$ is abnormal case. From the figures, we can see the two cases are roughly separated without depending on users, and this suggests that the CAD could be sufficiently applied to the CR images by the customization.

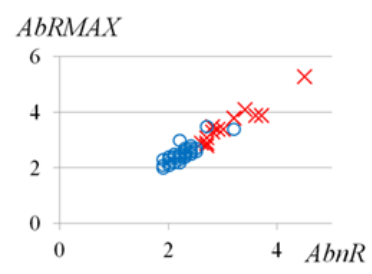

(a) UserA

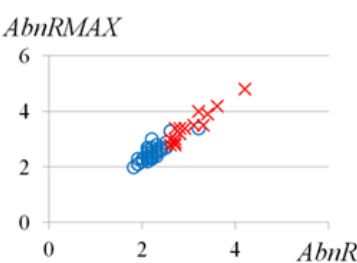

(b) User B

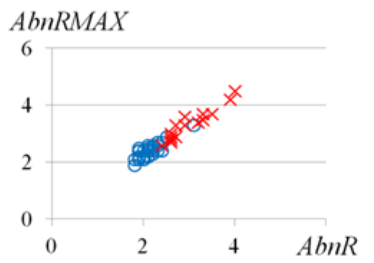

(c) User C

Figure 7: Distribution maps of all the image data by the abnormalities.

Figure 8 shows results of an image drawn by each user. From the results, we can see that there are difference of the drawing between the users. In addition, although there were no significant difference in the discrimination ratios shown in Table 2 between the users, it is suggested that there is possibility the abnormalities could be depended on the user if the drawing is depended on users. Therefore, it is necessary to reduce the difference between users by improving the manual more strictly.

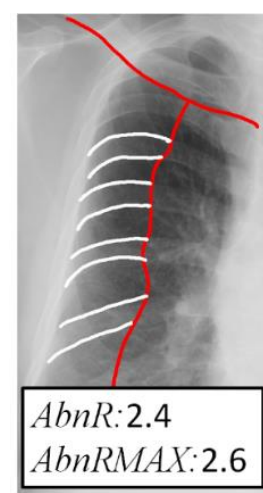

(a) User A

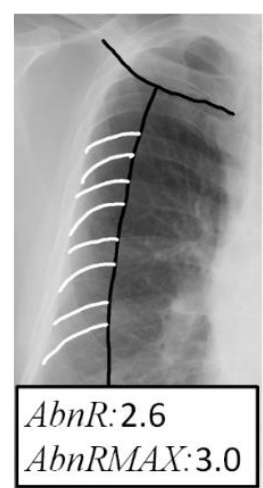

(b) User B

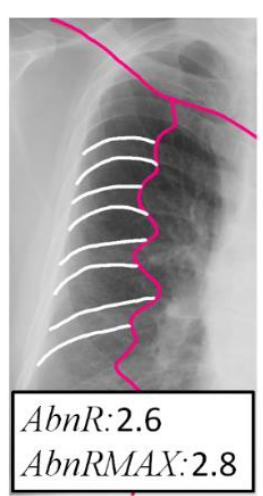

(c) User C

Figure 8: Results of the drawing for an image by the 3 users. 
Koji Abe, Masahide Minami, Ryosuke Miyazaki and Haiyan Tian; Application of a Computer-aid Diagnosis of Pneumoconiosis for CR X-ray Images, Journal of Biomedical Engineering and Medical Imaging, Volume 1, No 5, Oct (2014) ,pp 113-122

Finally, we compare experimental results of the discrimination between the proposed method and the existing CAD, i.e., the proposed method is the CAD which apples to the CR X-ray images by the customization of the rib drawing and the existing CAD works to the X-ray images digitalized by CCD scanner. Table 3 shows experimental results of the discrimination for the existing CAD using the same 51 $\mathrm{X}$-ray images digitalized by CCD scanner. Comparing Table 2 with Table 4, we can confirm there is no significant difference between the two CAD systems. In Japan, there is the license test of mammography, where the candidates have to correctly read the X-ray pictures of more than $80 \%$ to pass the test. If we follow this line, the results of Table 2 show that the CAD can be applied to the CR images sufficiently.

Table 3: Experimental results of the discrimination by the existing method (using images digitalized by CCD scanner).

\begin{tabular}{|c|c|c|c|c|c|c|c|}
\hline \multirow{2}{*}{ User } & \multirow{2}{*}{ Case } & \multicolumn{2}{|c|}{ RT } & \multicolumn{2}{c|}{ NN } & \multicolumn{2}{c|}{ SVM } \\
\cline { 3 - 8 } & & Rec. & Pre. & Rec. & Pre. & Rec. & Pre. \\
\hline \multirow{2}{*}{ userA } & Normal & $95.7 \%$ & $95.7 \%$ & $95.7 \%$ & $95.7 \%$ & $95.7 \%$ & $95.7 \%$ \\
\cline { 2 - 8 } & Abnormal & $83.3 \%$ & $83.3 \%$ & $83.3 \%$ & $83.3 \%$ & $83.3 \%$ & $83.3 \%$ \\
\hline \multirow{2}{*}{ userB } & Normal & $95.7 \%$ & $95.7 \%$ & $97.9 \%$ & $97.9 \%$ & $97.9 \%$ & $97.9 \%$ \\
\cline { 2 - 8 } & Abnormal & $83.3 \%$ & $83.3 \%$ & $91.7 \%$ & $91.7 \%$ & $91.7 \%$ & $91.7 \%$ \\
\hline \multirow{2}{*}{ userC } & Normal & $97.9 \%$ & $97.9 \%$ & $100.0 \%$ & $97.9 \%$ & $97.9 \%$ & $100.0 \%$ \\
\cline { 2 - 8 } & Abnormal & $91.7 \%$ & $91.7 \%$ & $91.7 \%$ & $100.0 \%$ & $100.0 \%$ & $92.3 \%$ \\
\hline
\end{tabular}

7 Conclusion

For the sake of application of the CAD system for pneumoconiosis for images digitalized by CCD scanner to chest CR X-ray images, this paper proposed a preprocessing of customizing the manual for drawing rib edges in the CAD. Experimental results for examining the proposed method using 51 chest CR X-ray images showed that the customization was effective enough for the diagnosis and there was no significant difference between the CAD which applied to the CR images and the existing CAD which works to the X-ray images digitalized by CCD scanner.

As future works, it is necessary to improve the manual more strictly to reduce difference of the drawing between users.

\section{REFERENCES}

[1]. R.P. Kruger, W.B. Thompson, and A.F. Turner, Computer diagnosis of pneumoconiosis. Trans. on Systems, Man. and Cybernetics, 1974. SMC-4(1): pp. 40-49.

[2]. H. Kobatake and K. Ohishi, Automatic diagnosis of pneumoconiosis by texture analysis of chest x-ray images. Proc. IEEE ICASSP, 1987. pp. 633-636.

[3]. A.M. Savol, C.C. Li, and R.J. Hoy, Computer aided recognition of small rounded pneumoconiosis opacities in chest X-rays. IEEE Transactions on PAMI, 1980. 2(5): pp. 479-482.

[4]. T. Kouda and H. Kondo, Computer-aided diagnosis for pneumoconiosis using neural network. Biomed. Soft Comput. Hum. Sci., 2001. 7(1): pp. 13-18. 
Journal of Biomedical Engineering and Medical Imaging, Volume 1, Issue 5, October, 2014

[5]. M. Nakamura, K. Abe, and M. Minami, Quantitative Evaluation of Pneumoconiosis in Chest Radiographs Obtained with a CCD Scanner. Proc. of the $2^{\text {nd }}$ International Conference on the Applications of Digital Information and Web Technologies (ICADIWT 2009), 2009. pp. 673-678.

[6]. K. Abe, T. Tahori, M. Minami, M. Nakamura, and H. Tian, Computer-aided diagnosis of pneumoconiosis X-ray images scanned with a common CCD scanner. Automation Control and Intelligent Systems, 2013. 1(2): pp. 24-33.

[7]. International Labour Organization (ILO), Guidelines for the use of ILO international classification of radiographs of pneumoconiosis (Revised edition 2011). Geneva: International Labour Office.

[8]. F. Mosteller, A k-sample slippage test for an extreme population. The Annals of Mathematical Statistics, 1948. 19(1): pp. 58-65. 